\title{
ac response of quantum point contacts with a split-gate configuration
}

\author{
Kenji Sasaoka, ${ }^{1,3, *}$ Takahiro Yamamoto, ${ }^{2, \dagger}$ Satoshi Watanabe, ${ }^{2}$ and Kenji Shiraishi ${ }^{1,3}$ \\ ${ }^{1}$ Center for Computational Sciences, University of Tsukuba, Tsukuba, Ibaraki 305-8577, Japan \\ ${ }^{2}$ Department of Materials Engineering, The University of Tokyo, 7-3-1 Hongo, Bunkyo, Tokyo 113-8656, Japan \\ ${ }^{3}$ CREST, Japan Science and Technology Agency, Chiyoda-ku, Tokyo 102-0075, Japan
}

(Received 17 December 2010; revised manuscript received 8 August 2011; published 2 September 2011)

\begin{abstract}
The alternating-current response of a quantum point contact (QPC) is numerically investigated using the nonequilibrium Green function method combined with an effective mass theory. We found that the susceptance of a QPC increases stepwise with increasing gate voltage, when the width of the quantization plateau in the gate voltage-conductance curve is narrower than the width of the region where the conductance changes gradually. We also show that the height of a susceptance step is proportional to the ac-bias frequency. These simulation results are in excellent qualitative agreement with recent experimental results. Moreover, we found that the transition from capacitive susceptance to inductive susceptance occurs with increasing gate voltage. The capacitive-inductive transition point is independent of the ac-bias frequency, but it does depend on the contact width.
\end{abstract}

DOI: 10.1103/PhysRevB.84.125403

PACS number(s): 73.23.-b, 73.63.Rt

\section{INTRODUCTION}

Electronic transport in quantum point contacts (QPCs) has been extensively investigated both experimentally and theoretically ${ }^{1,2}$ because QPCs are not only test systems for quantum transport but also potential candidates for basic elements of future nanoscale devices. ${ }^{3,4}$ Although there has been substantial progress for direct current transport in QPCs over the last two decades, little attention has been paid to the alternating current transport in QPCs. ${ }^{5-9}$ However, ac transport in QPCs is critical for designing and manipulating ultrafast operations in future QPC-based devices. ${ }^{10}$

Recently, Hohls et al. measured the admittance of QPCs at frequencies up to $300 \mathrm{MHz}{ }^{8,9}$ They formed a QPC in a GaAs/AlGaAs heterostructure by using a pair of metallic gate electrodes (known as the split-gate configuration). Their admittance data for the QPC exhibits four remarkable characteristic features: (i) the width of quantization plateaus seen in the change of the real part of the admittance (ac conductance) with increasing gate voltage is narrow and in most regions the ac conductance increases gradually; (ii) there is no notable difference between the ac conductance up to $300 \mathrm{MHz}$ and the dc conductance; (iii) the imaginary part of the admittance (the susceptance) has a stepwise variation with respect to the gate voltage; and (iv) the height of susceptance step is proportional to the frequency $v$ of the ac-bias voltage. In other words, the susceptance is a linear function of $v$ below $v=300 \mathrm{MHz}$. In addition to these four features, the following problem remains to be resolved: (v) due to experimental difficulties (e.g., the influence of parasitic admittance), it has not been clarified whether the susceptance changes its sign from positive (i.e., inductive response) to negative (i.e., capacitive response) at a certain gate voltage.

The ac response of QPCs has also been investigated theoretically by Büttiker ${ }^{5}$ and Aronov et al. ${ }^{6}$ They succeeded in reproducing some of the experimental observations of features (i)-(iv). Büttiker predicted features (ii) and (iv) using the Wentzel-Kramers-Brillouin (WKB) approximation. Aronov et al. also showed features (ii) and (iv) by solving the Boltzmann kinetic equation for QPCs in the form of a smooth constriction whose dimension is much longer than the wavelength (the adiabatic approximation). For point (v), they argue that the inductive-capacitive transition does occur at a certain gate voltage.

Although these theoretical studies succeeded in explaining two characteristic features, (ii) and (iv), the theoretical results are inconsistent with the other two features, (i) and (iii). More specifically, the calculated conductance exhibits well-developed conductance quantization and changes steeply between plateaus in quite narrow voltage ranges. In addition, although the calculated susceptance displays a stepwise structure, it has sharp peaks at the step edges, which were not observed in the experiment by Hohls et al. The appearance of sharp peaks in previous theoretical studies implies that the WKB approximation and the adiabatic approximation are broken at the susceptance step edges, as Büttiker already pointed out in Ref. 5. Thus, approaches besides the WKB and adiabatic approximations are required to reproduce the susceptance without the sharp peaks at the step edges. In this study, we adopt Ando's expression for a confinement potential formed by a split gate and the nonequilibrium Green function (NEGF) method as a numerical technique to calculate the admittance of QPCs whose dimensions are less than the electron wavelength. ${ }^{12}$ Based on these theoretical tools and keeping in mind the conditions under which the approximations used in previous studies ${ }^{5,6}$ are appropriate, we attempt to reproduce the characteristic features (i)-(iv) and to answer point (v).

This paper is organized as follows. In Sec. II, we briefly describe the effective-mass Hamiltonian of a QPC (Sec. II A) and give its discretized expression, which is required for numerical calculations (Sec. II B). Next, we briefly review the NEGF description of admittance (Sec. II C). Section III reports the simulation results for the admittance of a QPC. Finally, the paper is summarized in Sec. IV.

\section{MODEL AND METHOD}

\section{A. Effective-mass theory of quantum point contacts}

In this subsection, we briefly review the effective-mass theory of QPCs. ${ }^{13}$ The effective-mass Hamiltonian in two 

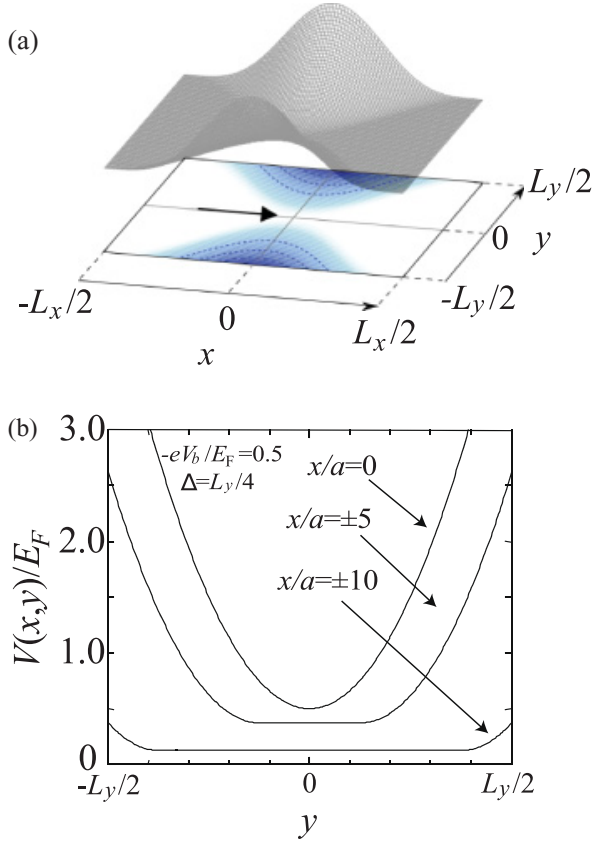

FIG. 1. (Color online) (a) Confinement potential of a QPC with a length $L_{x}=30 a$ and a width $L_{y}=30 a$ with $a=2.5 \mathrm{~nm}$. The arrow shows the direction of the electron flow. (b) Confinement potential profile for $-e V_{b} / E_{F}=0.5$ and $\Delta=L_{y} / 4$. The solid curves represent the potentials at $x / a=0, \pm 5$, and \pm 10 as a function of $y$.

dimensions is described by

$$
H=-\frac{\hbar^{2}}{2 m^{*}}\left(\frac{\partial^{2}}{\partial x^{2}}+\frac{\partial^{2}}{\partial y^{2}}\right)+V(x, y),
$$

where $m^{*}$ is the effective mass of a band electron and $V(x, y)$ represents the confinement potential of a QPC. In our simulation, we use Ando's expression for a confinement potential (Fig. 1), ${ }^{11}$ which is given by

$$
\begin{aligned}
V(x, y)= & -\frac{e V_{b}}{2}\left(1+\cos \left[\frac{2 \pi x}{L_{x}}\right]\right) \\
& +E_{F} \sum_{ \pm}\left(\frac{y-y_{ \pm}(x)}{\Delta}\right)^{2} \Theta\left(y^{2}-y_{ \pm}(x)^{2}\right),
\end{aligned}
$$

with

$$
y_{ \pm}(x)= \pm \frac{L_{y}}{4}\left(1-\cos \left[\frac{2 \pi x}{L_{x}}\right]\right),
$$

where $e(>0)$ is the elementary charge and $L_{x}$ and $L_{y}$ are, respectively, the length and width of the confinement regions of a QPC $\left(-L_{x} / 2 \leqslant x \leqslant L_{x} / 2\right.$ and $\left.-L_{y} / 2 \leqslant y \leqslant L_{y} / 2\right)$. $\Theta(t)$ represents the Heaviside step function [i.e., $\Theta(t)=1$ for $t>0$ and $\Theta(t)=0$ for $t<0]$. $\Delta$ determines the curvature of the confinement potential so that it modulates the contact width of the QPC. $E_{F}$ is the Fermi energy of the electrodes, and $V_{b}$ is the potential barrier height at $x=0$. The potential barrier is induced around the center of the QPC for $V_{b}<0$, while $V_{b}>0$ gives an attractive potential along the $x$ axis. Outside the confinement region, the potential is given by $V(x, y)=0$ in the electrode regions $\left(|x|>L_{x} / 2\right.$ and $\left.|y| \leqslant L_{y} / 2\right)$ and by $V(x, y)=\infty$ for $|y|>L_{y} / 2$.
For a fixed width $L_{y}$, the length $L_{x}$ determines the length scale of the system. The adiabatic limit used in the previous studies corresponds to $L_{x} \gg \lambda_{F} .^{5,6}$

The split gate mainly modulates the potential barrier height $V_{b}$, and the curvature of the confinement potential $\Delta$. Therefore, we examine the $V_{b}$ - and $\Delta$-dependences on the admittance of QPCs in Sec. III.

\section{B. Discretized expression for the effective-mass Hamiltonian}

To perform numerical calculations for the admittance of the QPC, we discretize the effective-mass Hamiltonian in Eq. (1) on a square lattice with a lattice constant $a$. Discrete lattice points are located at $x=n a$ and $y=m a$, where $n=$ $-\left(N_{x}-1\right) / 2,-\left(N_{x}-3\right) / 2, \cdots,\left(N_{x}-1\right) / 2\left(=L_{x} / 2 a\right)$ and $m=-\left(N_{y}-1\right) / 2,-\left(N_{y}-3\right) / 2, \cdots,\left(N_{y}-1\right) / 2\left(=L_{y} / 2 a\right)$. Thus, the effective-mass Hamiltonian in Eq. (1) is mapped onto a nearest-neighbor tight-binding Hamiltonian as

$$
\begin{aligned}
H= & \sum_{n, m} \epsilon_{n m}|n, m\rangle\langle n, m| \\
& -\sum_{\left\langle n, m ; n^{\prime}, m^{\prime}\right\rangle} t\left(|n, m\rangle\left\langle n^{\prime}, m^{\prime}\right|+\text { H.c. }\right),
\end{aligned}
$$

where $t=\hbar^{2} / 2 m^{*} a^{2}$ is the hopping energy and $\epsilon_{n m}=4 t+$ $V_{n m}$ is the onsite energy. The confinement potential in Eq. (2) is also discretized as

$$
\begin{aligned}
V_{n m}= & -\frac{e V_{b}}{2}\left(1+\cos \left[\frac{2 \pi n}{N_{x}-1}\right]\right) \\
& +E_{F} \sum_{ \pm}\left(\frac{m-y_{ \pm, n}}{\Delta / a}\right)^{2} \Theta\left(m^{2}-y_{ \pm, n}^{2}\right),
\end{aligned}
$$

where

$$
y_{ \pm, n}= \pm \frac{N_{y}-1}{4}\left(1-\cos \left[\frac{2 \pi n}{N_{x}-1}\right]\right) .
$$

In this paper, we focus on the QPC formed in a GaAs/AlGaAs heterostructure with electron density $n_{e}=5 \times$ $10^{11} \mathrm{~cm}^{-2}$. The lattice constant is chosen to be $a=2.5 \mathrm{~nm}^{14}$ and $L_{y}=30 a\left(N_{y}=31\right)$, both fixed throughout this study. The hopping parameter is estimated to be $t \approx 90 \mathrm{meV}$ using a typical effective mass of an electron in $\mathrm{AlGa} / \mathrm{AlGaAs}$ heterostructures, $m^{*}=0.067 m_{e}$, where $m_{e}$ is the free electron mass. A typical Fermi wavelength (Fermi energy) of electrons in $\mathrm{AlGa} / \mathrm{AlGaAs}$ heterostructures is given by $\lambda_{F}=35 \mathrm{~nm}$ $\left(E_{F}=20 \mathrm{meV}\right)$. Thus, $\lambda_{F}$ is much longer than the channel length $L_{x}$ in our simulation system $\left(L_{x} \ll \lambda_{F}\right)$. This is opposite to previous theoretical studies. 5,6

\section{Nonequilibrium Green function method}

In this subsection, we briefly describe the NEGF method for ac transport. It has been successfully applied to various mesoscopic ${ }^{15,16}$ and nanoscale systems. ${ }^{17-21}$ Within the linear response approximation with respect to the amplitude of the ac-bias voltage $V_{\beta}$, the electric current flowing from the QPC to the left ( $\alpha=\mathrm{L})$ or right $(\alpha=\mathrm{R}$ ) lead is expressed by

$$
I_{\alpha}(t)=\operatorname{Re}\left[I_{\alpha}(\omega) e^{-i \omega t}\right]
$$


with

$$
I_{\alpha}(\omega)=\sum_{\beta=\mathrm{L}, \mathrm{R}} Y_{\alpha \beta}(\omega) V_{\beta} .
$$

Here, $\omega$ is the ac-bias frequency and $Y_{\alpha \beta}(\omega)$ is the admittance between the $\alpha$-and $\beta$-leads; the admittance consists of two parts associated with the conduction current $Y_{\alpha \beta}^{c}(\omega)$ and the displacement current $P_{\alpha} Y_{\beta}^{d}(\omega)$ :

$$
Y_{\alpha \beta}(\omega)=Y_{\alpha \beta}^{c}(\omega)+P_{\alpha} Y_{\beta}^{d}(\omega),
$$

where $P_{\alpha}$ are the partition coefficients. These coefficients should satisfy the conditions $\sum_{\alpha} P_{\alpha}=1$ and $P_{\alpha}=$ $-\sum_{\gamma} Y_{\alpha \gamma}^{c}(\omega) / \sum_{\gamma} Y_{\gamma}^{d}(\omega)$, which are derived from current conservation $\left[\sum_{\beta} Y_{\alpha \beta}(\omega)=0\right]$ and gauge invariance [ $\left.\sum_{\alpha} Y_{\alpha \beta}(\omega)=0\right]$, respectively. ${ }^{22,23}$ These two requirements give a relation between the admittance elements:

$$
Y(\omega) \equiv Y_{\mathrm{LR}}(\omega)=Y_{\mathrm{RL}}(\omega)=-Y_{\mathrm{LL}}(\omega)=-Y_{\mathrm{RR}}(\omega) .
$$

The conductance [the real part of $Y(\omega)$ ] is an even function with respect to $\omega$, and it is always positive, whereas the susceptance [the imaginary part of $Y(\omega)$ ] is an odd function with respect to $\omega$ and it can be both positive and negative. In our convention for the sign of the phase difference between the current and the acbias voltage, positive and negative susceptances, respectively, indicate inductive and capacitive susceptances. Note that this convention is opposite to that commonly employed in electric circuit theory.

The conduction current admittance $Y_{\alpha \beta}^{c}(\omega)$ and the displacement current admittance $Y_{\beta}^{d}(\omega)$ are expressed within the NEGF formulation under the wide-band limit, where the energydependent self-energy is replaced with the energy-independent self-energy: $\boldsymbol{\Sigma}_{\alpha}(\epsilon) \rightarrow \boldsymbol{\Sigma}_{\alpha}\left(E_{\mathrm{F}}\right){ }^{12}$ The conduction-current admittance $Y_{\alpha \beta}^{c}(\omega)$ is expressed by

$$
Y_{\alpha \beta}^{c}(\omega)=\frac{2 e^{2}}{h} \int_{-\infty}^{\infty} d \epsilon \frac{f_{\alpha}(\epsilon)-f_{\alpha}(\epsilon+\hbar \omega)}{\hbar \omega} T_{\alpha \beta}(\epsilon, \omega),
$$

where $f_{\alpha}(\epsilon)$ is the Fermi-Dirac distribution function of the $\alpha$ lead, and the factor 2 in Eq. (11) is the spin degrees of freedom. $T_{\alpha \beta}(\epsilon, \omega)$ is the effective transmission function under an ac-bias voltage with frequency $\omega$, which is given by

$$
\begin{aligned}
T_{\alpha \beta}(\epsilon, \omega)= & \operatorname{Tr}\left[\boldsymbol{G}(\epsilon+\hbar \omega) \boldsymbol{\Gamma}_{\beta} \boldsymbol{G}^{\dagger}(\epsilon) \boldsymbol{\Gamma}_{\alpha}\right] \\
& -i \delta_{\alpha \beta} \operatorname{Tr}\left[\boldsymbol{G}(\epsilon+\hbar \omega) \boldsymbol{\Gamma}_{\alpha}-\boldsymbol{\Gamma}_{\alpha} \boldsymbol{G}^{\dagger}(\epsilon)\right] .
\end{aligned}
$$

Here, $\boldsymbol{G}(\epsilon)=\left[(\epsilon+i \eta) \boldsymbol{I}-\boldsymbol{H}-\sum_{\alpha} \boldsymbol{\Sigma}_{\alpha}\right]^{-1}$ is the retarded Green function, where $\eta$ is a positive infinitesimal and $\boldsymbol{I}$ is the identity matrix. $\boldsymbol{H}$ is the matrix representation for the discretized effective-mass Hamiltonian (4) in the confinement region of a QPC $\left(|x| \leqslant L_{x} / 2,|y| \leqslant L_{y} / 2\right)$. The self-energy due to the $\alpha$ lead can be analytically expressed as ${ }^{13}$

$$
\boldsymbol{\Sigma}_{\alpha}\left(E_{F}\right)=\sum_{l=1}^{N_{y}} \frac{E_{F}-\epsilon_{l}-\sqrt{\left(E_{F}-\epsilon_{l}\right)^{2}-4 t^{2}}}{2} \boldsymbol{u}_{l} \boldsymbol{u}_{l}^{\dagger}
$$

Here, $\epsilon_{l}$ and $\boldsymbol{u}_{l}$ are, respectively, given by

$$
\epsilon_{l}=-2 t \cos \frac{\pi l}{N_{y}+1}+4 t .
$$

and

$$
u_{l, m}=\sqrt{\frac{2}{N_{y}+1}} \cos \frac{\pi l}{N_{y}+1} m,
$$

where $m=-\left(N_{y}-1\right) / 2,-\left(N_{y}-3\right) / 2, \cdots,\left(N_{y}-1\right) / 2$.

The level broadening function $\boldsymbol{\Gamma}_{\alpha}$ is obtained from the relation $\boldsymbol{\Gamma}_{\alpha}=-2 \operatorname{Im} \boldsymbol{\Sigma}_{\alpha}$. In the dc limit $(\omega \rightarrow 0)$, the conduction current admittance expression in Eq. (11) reduces to the Landauer formula for dc transport. ${ }^{13}$ On the other hand, the displacement current admittance $Y_{\beta}^{d}(\omega)$ is described by

$$
Y_{\beta}^{d}(\omega)=\frac{2 e^{2}}{\hbar} \int_{-\infty}^{\infty} d \epsilon\left[f_{\beta}(\epsilon)-f_{\beta}(\epsilon+\hbar \omega)\right] Y_{\beta}^{d}(\epsilon, \omega),
$$

where the energy-resolved admittance $Y_{\beta}^{d}(\epsilon, \omega)$ is expressed by

$$
Y_{\beta}^{d}(\epsilon, \omega)=-i \operatorname{Tr}\left[\boldsymbol{G}(\epsilon+\hbar \omega) \boldsymbol{\Gamma}_{\beta} \boldsymbol{G}^{\dagger}(\epsilon)\right],
$$

in terms of Green functions. The displacement current admittance in Eq. (10) vanishes, $Y_{\beta}^{d}(\omega)=0$, in the zero-frequency limit. In the NEGF formalism combined with the current partition of Eq. (9), ${ }^{23}$ the displacement current admittance vanishes when no electrons transfer between a lead and the QPC, i.e., $Y_{\beta}^{d}(\omega)=0$ for $\boldsymbol{\Gamma}_{\beta}=0$. This means that only the charge accumulation due to the electron transfer is taken into account in this treatment, while the accumulation due to the long-range Coulomb interaction is not. This treatment is valid in the limit of weak Coulomb interactions, such as considered in the present work. On the other hand, when effects of the long-range Coulomb interaction in the admittance are not negligible, discussion of displacement current, taking into account a solution of the Poisson equation(or a geometrical capacitance as a simplified representation), ${ }^{24}$ is necessary, instead of the current partition as described in Eq. (9).

In our numerical simulation, the Green functions were calculated using a recursive method. ${ }^{25}$

\section{NUMERICAL RESULTS}

In this section, we present the numerical results for the ac response of the QPC obtained by the NEGF simulation. As mentioned in Sec. II A, the split-gate voltage modulates the potential barrier height $V_{b}$ and the potential curvature $\Delta$ of the confinement potential in Eq. (2). In Sec. III A, we discuss the $V_{b}$ dependence of the ac response of the QPCs by comparing our simulation results with experimental results and previous theoretical results. In Sec. III B, we explain the $\Delta$ dependence of the ac response of the QPC, focusing particularly on the transition between the capacitive susceptance and the inductive susceptance.

\section{A. Barrier-height dependence of ac response}

Figures 2(a) and 2(b), respectively, show the $V_{b}$-dependence of the conductance and the susceptance of the QPC with a fixed $\Delta=L_{y} / 4$ and $L_{x}=30 a$ at zero temperature. As seen in Fig. 2(a), the conductance of the QPC exhibits only small and partially formed quantization plateaus, and changes continuously in most of the region as a function of the height of barrier potential. This is because the Fermi wavelength 

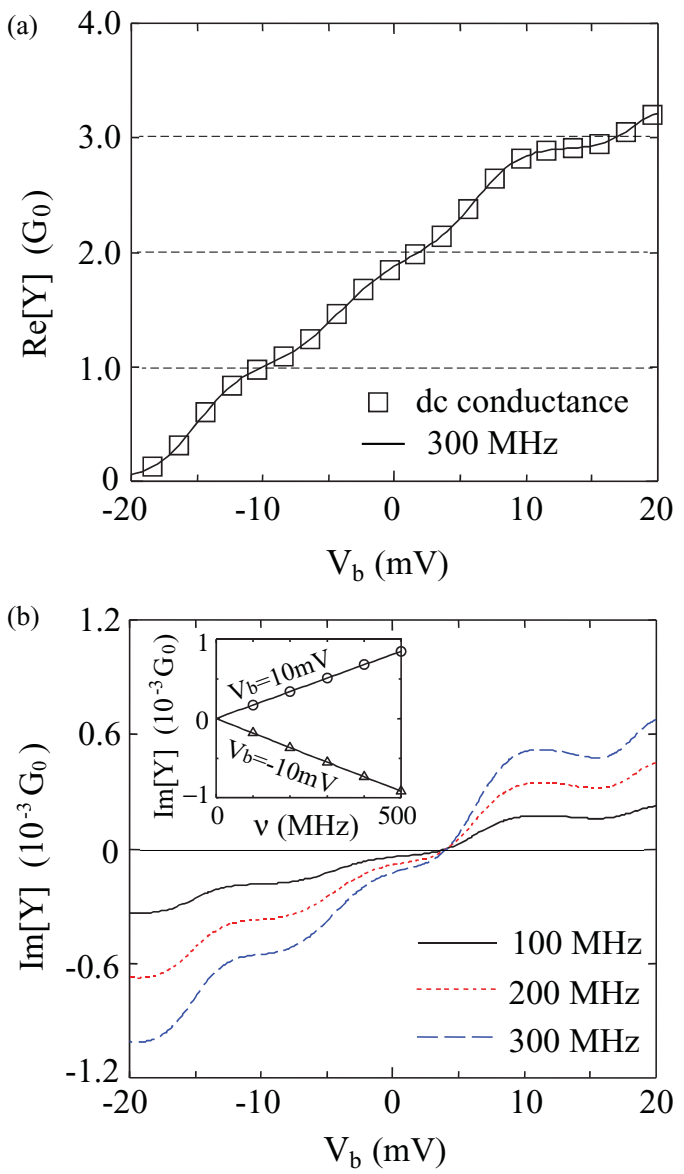

FIG. 2. (Color online) (a) Conductance and (b) susceptance of a QPC as a function of the height of the potential barrier induced by the gate voltage $V_{b}$. The solid, dotted, and dashed curves correspond to ac-bias frequencies of $v=100,200$, and $300 \mathrm{MHz}$, respectively. Open squares in (a) denote the dc conductance. The inset shows the ac-bias frequency dependence of the susceptance for susceptance steps of $V_{b}=10 \mathrm{mV}$ (circles) and $-10 \mathrm{mV}$ (triangles). The step edge height is proportional to the ac-bias frequency in the low-frequency regime that is shown in the inset.

$\lambda_{F}$ of a GaAs/AlGaAs heterostructure is typically much longer than the QPC length $L_{x}$ in the present simulation (see Sec. II B). This conductance behavior is consistent with the experimental data obtained by Hohls et al., ${ }^{8,9}$ whereas it differs from previous theoretical results obtained under the condition $\lambda_{F} \ll L_{x}$ imposed by the $\mathrm{WKB}^{5}$ and adiabatic approximations. ${ }^{6}$

In addition, there is no notable difference between the conductances under $v=300 \mathrm{MHz}$ (solid curve) and the dc conductance (open squares) in Fig. 2(a). This implies that the ac-bias frequency, $v=300 \mathrm{MHz}$, is sufficiently low to neglect the frequency dependence of the conductance. This result is consistent with experimental data ${ }^{8,9}$ as well as previous theoretical results. ${ }^{5,6}$

As Fig. 2(b) shows, the susceptance also depends on the barrier height $V_{b}$. Here, the solid, dotted, and dashed curves correspond to ac-bias frequencies $\nu=\omega / 2 \pi=100$, 200 , and $300 \mathrm{MHz}$, respectively. The susceptance exhibits a stepwise structure with respect to $V_{b}$. In contrast to previous theoretical studies, ${ }^{5,6}$ the susceptances in Fig. 2(b) do not exhibit sharp peaks at the step edges corresponding to van Hove singularities. The unphysical susceptance peaks disappear due to the wavelength of an electron flowing through the QPC being much longer than the channel length of the QPC at van Hove singularities with zero wave number $k=0$. That is, the $\mathrm{WKB}^{5}$ and adiabatic approximations ${ }^{6}$ are unsuitable for describing the behavior of susceptance edges, as Büttiker states in Ref. 5.

Another important phenomenon is dependence of the susceptance on the ac-bias frequency $v$, which is in sharp contrast with the conductance in Fig. 2(a). The inset of Fig. 2 shows the $v$ dependences of the susceptance at $V_{b}=10 \mathrm{mV}$ (circles) and $-10 \mathrm{mV}$ (triangles). The susceptance at $V_{b}=10 \mathrm{mV}$ increases linearly with $v$, whereas that at $V_{b}=-10 \mathrm{mV}$ decreases linearly with $v$. The linear dependence of the susceptance on $v$ implies that $300 \mathrm{MHz}$ is sufficiently low to neglect higher-order components of the susceptance with respect to $v$. The linear dependence of the susceptance on $v$ implies that $300 \mathrm{MHz}$ is sufficiently low to neglect higher-order components of the susceptance with respect to $v$. This coefficient corresponds to the emittance $E$, which was originally defined in terms of the partial density of states. ${ }^{24,26,27}$ This is also consistent with previous experimental observations. ${ }^{8,9}$ The difference between the signs of the susceptances at $V_{b}= \pm 10 \mathrm{mV}$ is important in connection with point (v) mentioned in the introduction. Thus, our answer to the problem (v) is the same as the theoretical arguments given by Büttiker ${ }^{5}$ and Aronov et al. ${ }^{6}$ As a result of our work, we found from Fig. 2(b) that the inductive-capacitive transition point is independent of the ac-bias frequency $v$. The question now arises: what does the IC transition point depend on? In the next subsection, we show that it depends on the curvature of the confinement potential.

\section{B. Effects of confinement-potential curvature on ac response}

The curvature of the confinement potential is expressed by the parameter $\Delta$ in Eq. (2). Figures 3(a) and 3(b), respectively, display the conductance and susceptance for $\Delta=L_{y} / 4$ (solid curve), $L_{y} / 5$ (dotted curve), and $L_{y} / 6$ (dashed curve) for $v=$ $300 \mathrm{MHz}$, where $L_{x}=L_{y}=30 a$ with $a=2.5 \mathrm{~nm}$.

As Fig. 3(a) shows, the conductances decrease monotonically with decreasing $\Delta$. This means that the electron transmission decreases with decreasing contact width. This can be understood from the local density of states (LDOS) at the center $(x=0)$ of the QPC. It is intuitively obvious that the LDOS decreases with decreasing contact width, as shown in the inset of Fig. 3(a). Due to the reduction in the LDOS, the electron transmission decreases as $\Delta$ decreases.

Next, we focus on the $\Delta$ dependence of the susceptance, particularly of the IC transition point. The emittance increases with decreasing $\Delta$ for a fixed barrier height $V_{b}$, i.e., the split gate voltage adds a capacitive contribution to the emittance even when the geometrical capacitance is not taken into account. If the geometrical capacitance is taken into account, a stronger capacitive contribution to the emittance is expected with decreasing $\Delta .{ }^{28}$

The IC transition point moves toward higher $V_{b}$ as $\Delta$ decreases. However, the IC transition occurs immediately after the conductance exceeds $2 G_{0}$ for any value of $\Delta$. This critical conductance value corresponds to half the number of energy 

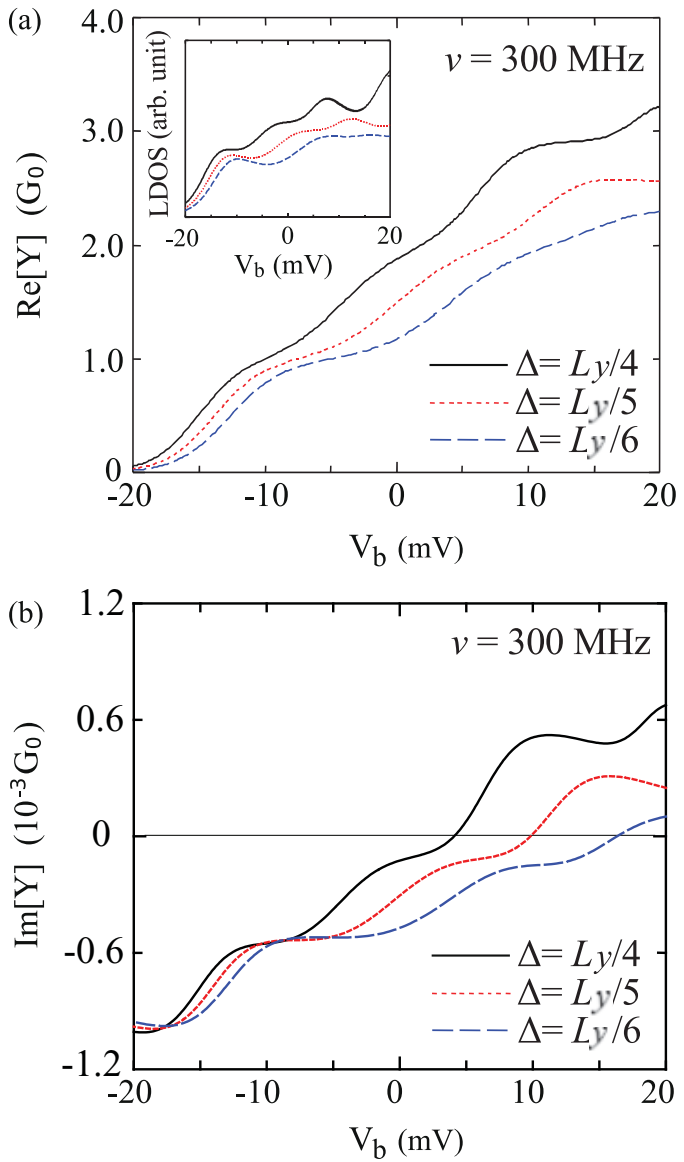

FIG. 3. (Color online) (a) Conductance and (b) susceptance of a QPC with $\Delta=L_{y} / 4$ (solid curve), $L_{y} / 5$ (dotted curve), and $L_{y} / 6$ (dashed curve) as a function of the gate voltage. Here, the ac-bias frequency is fixed at $v=300 \mathrm{MHz}$.

bands of the electrode crossing the Fermi level $E_{F}=20 \mathrm{meV}$. In the present models, the conduction channels are almost mutually independent, and the left and the right leads are connected symmetrically. Therefore, we can consider the IC transition to be similar to that in a double-barrier quantum well. Since the emittance $E$ is expected to behave as $E \propto$ $R-T,{ }^{5,20}$ where $R$ and $T$ are the reflection and transmission probabilities, respectively, the critical conductance value at the IC transition is $2 G_{0}$ irrespective of $\Delta$ and $E_{F}$ in the present models. It should be noted, however, that in general the critical conductance value depends on the Fermi level and the electrode structure.

Finally, we show the dependence of admittance on the height of the potential barrier for the QPC with $L_{x}=30 a$ (solid curve) and $L_{x}=50 a$ (dotted curve) at $\Delta=L_{y} / 6 a$, $L_{y}=30 a$, and $v=300 \mathrm{MHz}$ in Fig. 4. Here, the increase of the contact length corresponds to a smaller curvature of the confinement potential along the direction of the electron path through the QPC. As seen in Fig. 4(a), the conductance quantization plateaus appear more clearly with increasing QPC length, while the conductance transition becomes narrower. On the other hand, the susceptance continues to show a stepwise structure, although with inclined steps [see Fig. 4(b)]. This inclination comes from the small increase in susceptance at $V_{b}$ corresponding to the step edges, which correspond to the van
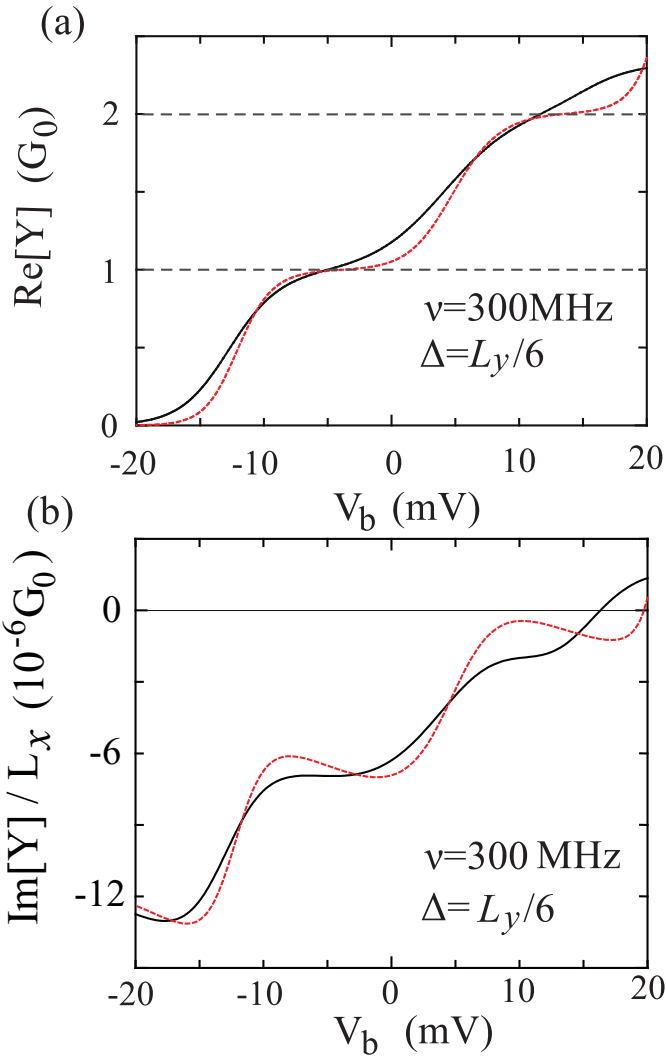

FIG. 4. (Color online) Dependence of the gate voltage on (a) the conductance and (b) susceptance of the QPC with $L_{x}=30 a$ (solid curve) and $L_{x}=50 a$ (dotted curve). Here, the ac-bias frequency and the potential curvature are fixed at $v=300 \mathrm{MHz}$ and $\Delta=L_{y} / 6$, respectively. The left and right axes in (b) are shown on the susceptance in the case of $L_{x}=30 a$ and of $L_{x}=50 a$.

Hove singularities. It is worth noting that the behavior of the susceptance for $L_{x}=50 a$ is much closer to the one obtained in previous theoretical studies, ${ }^{5,6}$ than that for $L_{x}=30 a$. This is because the QPC length of $L_{x}=50 a$ is comparable to the Fermi wavelength, ${ }^{29}$ and therefore the approaches of the previous studies are valid.

\section{CONCLUSION}

We performed numerical simulations of the ac response of a QPC using the NEGF method combined with a discretized effective-mass Hamiltonian. We describe QPCs using the confinement potential first proposed by Ando. ${ }^{11} \mathrm{We}$ found that the geometry of the QPC strongly influences both the dc- and ac-response behaviors. In contrast to the ac-response behavior of quantum wires with lengths longer than a typical electron wavelength, the susceptance of QPCs increases stepwise while the conductance changes gradually between the quantization plateaus with changes in gate voltage, and the region of this gradual change is broader than the plateau region. Moreover, the susceptances calculated in this study did not exhibit sharp peaks at the step edges corresponding to van Hove singularities of the energy band structure of the electrodes, in contrast with the sharp peaks observed in previous studies. ${ }^{5,6} \mathrm{We}$ also found that the QPCs undergo a transition from capacitive 
susceptance to inductive susceptance with increasing barrier height of the confinement potential. The inductive-capacitive transition point is independent of the ac-bias frequency, but it is sensitive to the curvature of the confinement potential. Thus, we succeeded in explaining characteristic features (i)-(iv) of recent experimental data for a QPC formed in a GaAs/AlGaAs heterostructure, ${ }^{8,9}$ and we predicted the IC transition of QPCs. In addition, the simulation method used in this paper can be widely applied to calculate the ac characteristics of various mesoscopic and nanoscale systems in addition to a QPC formed in a GaAs/AlGaAs heterostructure.

\section{ACKNOWLEDGMENTS}

We would like to thank Akira Sakai (Kyoto University) for valuable discussions on the ac-response measurement of QPCs. We also acknowledge partial financial support from Grants-in-Aid for Scientific Research on Innovative Areas "Materials Design through Computics: Complex Correlation and Non-Equilibrium Dynamics (2203)" (Grant No. 20104007) and for Scientific Research (Grant No. 20360016). Some of the numerical calculations were performed at the Supercomputer Center of the Institute for Solid State Physics, The University of Tokyo, Japan.
*Present address: Department of Materials Engineering, The University of Tokyo, 7-3-1 Hongo, Bunkyo, Tokyo 113-8656, Japan.

${ }^{\dagger}$ Present address: Department of Liberal Arts (Physics), Faculty of Engineering, Tokyo University of Science, Tokyo 102-0073, Japan. ${ }^{1}$ C. W. J. Beenakker and H. van Houten, Solid State Phys. 44, 1 (1991).

${ }^{2}$ H. van Houten and C. W. J. Beenakker, Phys. Today 49, 22 (1996).

${ }^{3}$ D. P. E. Smith, Science 269, 371 (1995).

${ }^{4}$ J. M. Elzerman, R. Hanson, L. H. W. van Beveren, B. Witkamp,

L. M. K. Vandersypen, and L. P. Kouwenhoven, Nature (London) 430, 431 (2004).

${ }^{5}$ T. Christen and M. Büttiker, Phys. Rev. Lett. 77, 143 (1996).

${ }^{6}$ I. E. Aronov, N. N. Beletskii, G. P. Berman, D. K. Campbell, G. D. Doolen, and S. V. Dudiy, Phys. Rev. B 58, 9894 (1998).

${ }^{7}$ J. Gabelli, G. Fève, T. Kontos, J.-M. Berroir, B. Placais, D. C. Glattli, B. Etienne, Y. Jin, and M. Büttiker, Phys. Rev. Lett. 98, 166806 (2007).

${ }^{8}$ J. Regul, F. Hohls, D. Reuter, A. D. Wieck, and R. J. Haug, Physica E 22, 272 (2004).

${ }^{9}$ F. Hohls, C. Fricke, and R. J. Haug, Physica E 40, 1760 (2008).

${ }^{10}$ S. Kang, C. Rutherglen, N. Rouhi, and P. Burke, IEEE Sensors Journal 10, 391 (2010).

${ }^{11}$ T. Ando, Phys. Rev. B 44, 8017 (1991).

${ }^{12} \mathrm{H}$. Haug and A. P. Jauho, Quantum Kinetics in Transport and Optics of Semiconductors (Springer, Berlin, 1996).

${ }^{13}$ S. Datta, Electronic Transport in Mesoscopic Systems (Cambridge University Press, Cambridge, England, 1995).

${ }^{14}$ A. Cresti, Phys. Status Solidi 203, 1172 (2006).
${ }^{15}$ M. P. Anantram and S. Datta, Phys. Rev. B 51, 7632 (1995).

${ }^{16}$ M. K. Yip, W. C. Kwok, J. Wang, and H. Guo, J. Appl. Phys. 89, 1777 (2001).

${ }^{17}$ C. Roland, M. Buongiorno Nardelli, J. Wang, and H. Guo, Phys. Rev. Lett. 84, 2921 (2000).

${ }^{18}$ D. Kienle and F. Léonard, Phys. Rev. Lett. 103, 026601 (2009).

${ }^{19}$ T. Yamamoto, K. Sasaoka, S. Watanabe, and K. Watanabe, Phys. Rev. B 81, 115448 (2010).

${ }^{20}$ T. Yamamoto, K. Sasaoka, and S. Watanabe, Phys. Rev. B 82, 205404 (2010).

${ }^{21}$ Z. Chen, J. Zhang, and Z. Yu, J. Comput. Theor. Nanosci. 7, 2331 (2010).

${ }^{22}$ M. Büttiker, A. Prêtre, and H. Thomas, Phys. Rev. Lett. 70, 4114 (1993).

${ }^{23}$ B. Wang, J. Wang, and H. Guo, Phys. Rev. Lett. 82, 398 (1999).

${ }^{24}$ M. Büttiker, J. Phys.: Condens. Matter. 5, 9361 (1993).

${ }^{25}$ D. J. Thouless and S. Kirkpatrick, J. Phys. C 14, 235 (1981).

${ }^{26}$ V. Gasparian, T. Christen, and M. Büttiker, Phys. Rev. A 54, 4022 (1996).

${ }^{27}$ T. Gramespacher and M. Büttiker, Phys. Rev. B 56, 13026 (1997).

${ }^{28} \mathrm{M}$. Büttiker and T. Christen, in Theory of Transport Properties of Semiconductor Nanostructures, edited by E. Scholl (Chapman and Hall, London, 1998).

${ }^{29}$ The Fermi wavelength $\lambda_{F} \sim 35 \mathrm{~nm}$ is almost equal to a quarter of the length of the QPC, $L_{x}=50 a \sim 125 \mathrm{~nm}$, which is the distance between the center of the QPC and the position with a half of the QPC width $\left(y_{+}-y_{-}=L_{y} / 2\right)$. 